Editorial

\title{
2006 International symposium on biomedical engineering, Taiwan (2006-ISOBME)
}

Biomedical engineering is a very significant research field which bridges the gap between the fundamental engineering knowledge and technology, and clinical practice and application. It includes a broad range of topics, both established and groundbreaking, from biomaterials to tissue engineering and cell engineering; from biomechanics to biomedical physics; from biomedical electronics to biomedical optoelectronics; from medical imaging to biomedical informatics. The growing research fruits of biomedical engineering assist in the better improvement of human health care.

This special issue arises out of a symposium entitled "2006 International symposium on biomedical engineering, Taiwan (2006-ISOBME)", held at the National Taiwan University in Taipei, Taiwan from December 14th to 16th 2006. The aims of the 2006-ISOBME were to explore and improve the research on biomedicine and health care, and to encourage greater involvement in its application with the main theme "Advances in Health Care Technology". This Special Issue provides the latest investigative results presented at the 2006-ISOBME and reflects the state-of-the-art of biomedical engineering research. Ten full length manuscripts selected cover areas including biomaterials, biomechanics, biomedical electronics, medical imaging, and tissue engineering. We hope that the Special Issue opens a way to understand what the clinicians and scientists in Taiwan contribute to the research field of biomedical engineering.

In compiling this special issue we were assisted by a committed Editorial Board and Review Panel, to whom we are extremely grateful. We expect that the large diffusion of this Special Issue will constitute an interesting point of departure for subsequent debate by a larger research community. We also hope you will enjoy these articles and find them stimulating.

\author{
Professor \\ Feng-Huei Lin (Ph.D.) ${ }^{\mathrm{a}, \mathrm{b}}$ \\ ${ }^{\text {a }}$ Congress chair, 2006-ISOBME, Taiwan \\ ${ }^{\mathrm{b}}$ Institute of Biomedical Engineering, \\ National Taiwan University, Taipei, Taiwan
}

